\title{
Recent Trends in SCADA Power Distribution Automation Systems
}

\author{
Hari Kumar Naidu ${ }^{a^{*}}$ and K. Thanushkodi ${ }^{\mathrm{b}}$ \\ ${ }^{a}$ Adhiyamaan college of Engineering, Hosur, T.N., India ${ }^{b}$ C.I.E.I.T. Coimbatore, T.N., India and Amtech \\ Automation, Bangalore, India.
}

\begin{abstract}
The objective of the study in this paper is to evaluate the advances in telecommunication, Information Technology and networking which offer SCADA Power supply Distribution automation as a solution to improve power distribution efficiencies. This paper also discusses the result of the indigenously developed prototype hardware and software model utilising the latest embedded technology innovation for SCADA Power Distribution Automation Systems for reliable performance of power system.
\end{abstract}

Keywords: SCADA, Distribution management system, GIS, Distribution automation, Embedded system.

\section{Introduction}

The Power Industry is in the midst of a transformation process from being an Energy suppliers to becoming an Energy Service Companies. The Distribution Utilities face new challenges every day and have to improve productivity by reducing operating and maintenance costs, while providing customers with a reliable power supply and a broad range of services.

The demand-supply gap in power in India is expected to grow by at least twenty per cent this year. The trend is expected to continue in the near future with no significant generating capacity coming up in the country and this may cause power crisis. It is imperative that power utilities need to look at increasing efficiencies in distribution networks, which are among the highest transmission and distribution losses in the world close to around 30 per cent. In addition, the social pricing for rural and other sectors increases pressure on utilities to improve productivity by reducing operating and maintenance costs to remain financially viable. To achieve rapid success, the effective use of Information Technology is essential. Distribution Management System is a tool for enterprise-wide management of an electric utility system for efficient operations, which enhances operational outputs and translates it into economic benefits. Some of the initiatives in distribution management include complete distribution automation, City power distribution automation, and SCADA based Power Distribution Automation Systems (KEMA, Ahmed and Soo, 2008; Antila et al., 2003;
Roytelman et al., 1993; Nenad Katic, 1995; Vladimir, 1995; Nenad Katie, 1998; Dragan, 2001)

\section{Need to automate}

Existing distribution systems have certain inherent inefficiencies due to their legacy. Firstly, most systems are monitored manually and results in maintenance taking place only during breakdowns. The present system also do not ensure reliable and complete power system usage information that can facilitate trend forecasting or help the utility in better analysis and planning. New challenges are faced by utilities due to the deregulation of the energy markets, greater environmental concern and the proliferation of open information systems. The new energy context requires revised strategies to confirm one's position as a prime power utility from management context as shown in figure1.

\section{SCADA systems}

Internationally, the power generation, the power transmission and the distribution attract equal investments. In India, in the last couple of years, utilities have started investing increasingly in various distribution automation tools for both cost reduction and service benefits.

One major tool available for power utilities is the Supervisory Control And Data Acquisition system. SCADA refers to a system that enables an electric utility to remotely

\footnotetext{
* Corresponding author: E-mail: cghhar@yahoo.co.in
} 


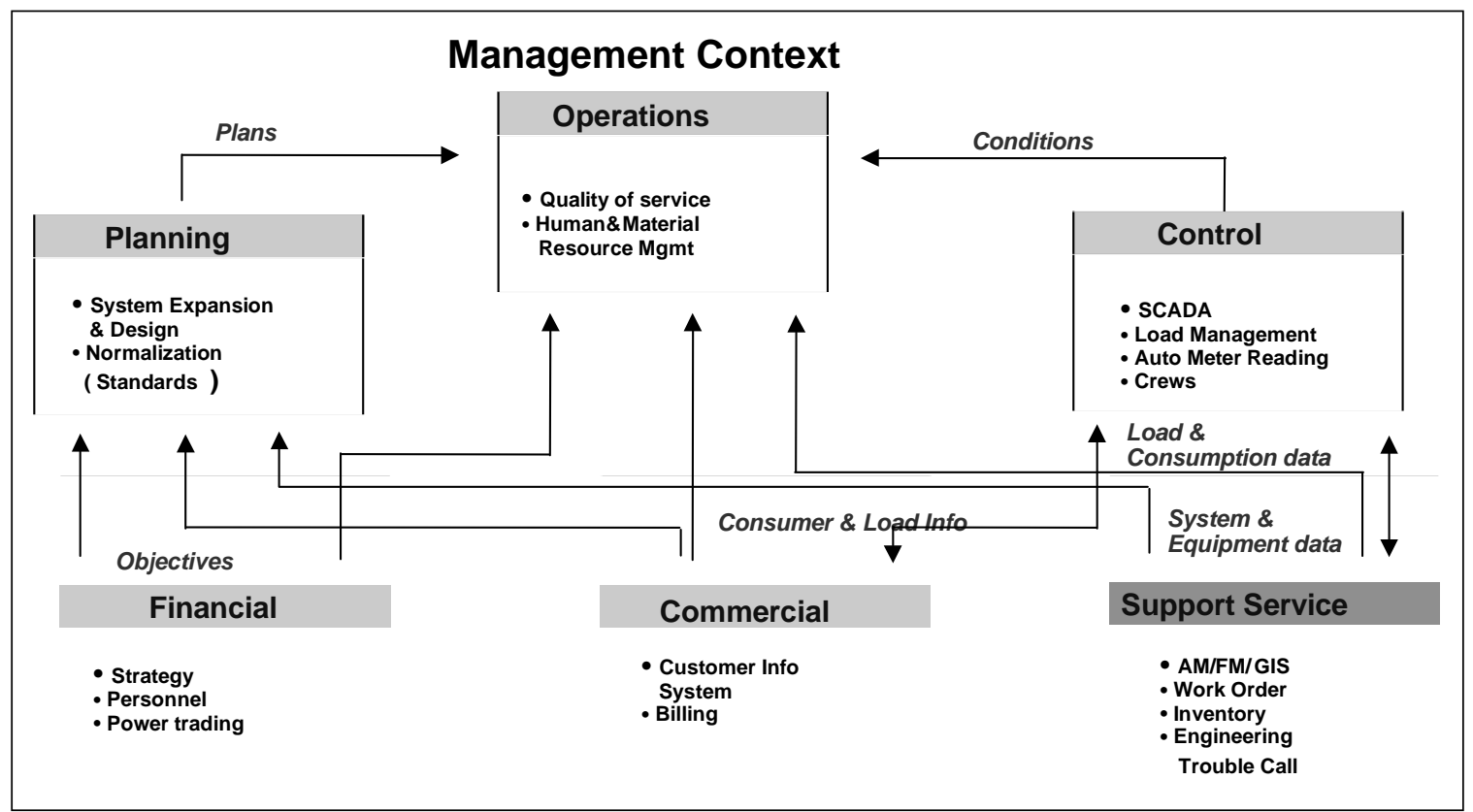

Fig. 1. Management context

monitor, coordinate, control and operate distribution components, equipment and devices in a real-time mode from remote locations with acquisition of data for analysis, and planning from one central location.

Today, SCADA systems monitor and control its distribution network. Distribution automation through SCADA systems directly leads to increased reliability of power to the consumers and results in lowering operating costs for the utility, forecasting accurate demand and supply management; faster restoration of power in case of a downturn and a quick alternate arrangement for power for important/emergency locations as shown in figure 2.

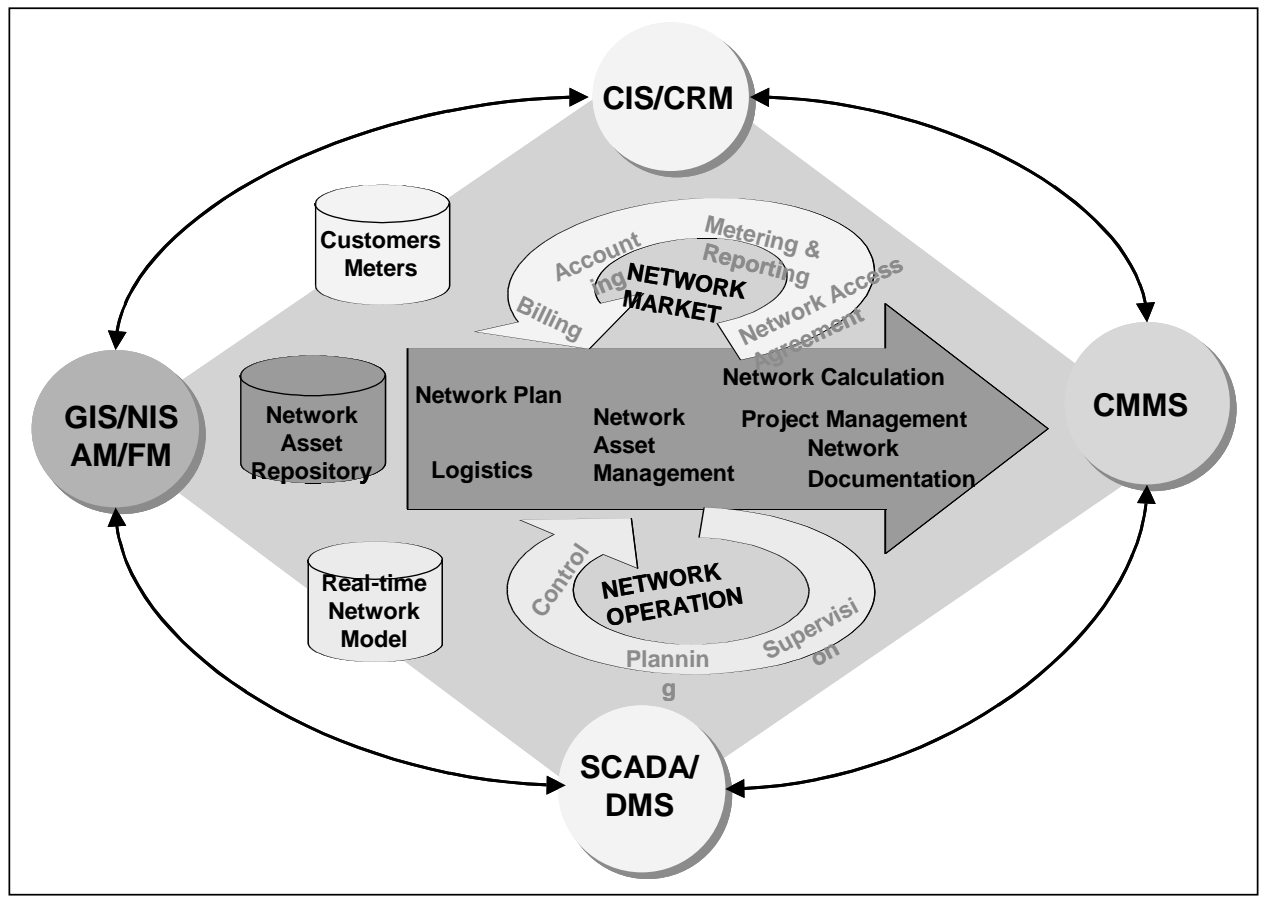

Fig. 2. Enterprise wide SCADA and Distribution Management System 


\section{Automation for both cost and service benefits:}

These benefits accumulate in areas related to investments, interruptions, and customer service as well as in areas related to operational savings.

\section{Economic benefits:}

Investment related benefits of Distribution Automation are derived from a more effective usage of the system. Distribution Automation provide increased availability of better data for planning, engineering and maintenance and result into capital deferred benefits. Lower operating costs are another major benefit of Distribution Automation achieved through improved voltage profiles, curtailed Voltage Automation Regulation flows, repair and maintenance savings, reduced feeder primary and distribution transformer load losses and load management.

\section{Improved reliability:}

On the qualitative side, improved reliability adds to the perceived value to the customers due to Volt-VAR control, loads balancing, Trouble call management, Emergency load shedding features of the state of the art Distribution Automation systems (Katic, 2002).

\section{Compatibility:}

Distribution Automation spans many functional and product areas including Computer systems, application software, Remote Terminal Units (RTUs), Communication systems and metering products as shown in figure3.

\section{Urban Systems}

The advances in communication technology, computer hardware and software, open system architecture, microprocessor based field devices, intelligent electronic devices (Remote Terminal Units) have completely changed the dynamics and utility of present day SCADA system as compared to the systems which were in existence only a few years ago. Today a field data communication device (RTU) can itself log on to the internet, upload the data to the host computer and receive tele-commands through the net. Added to this, the open architecture design of the

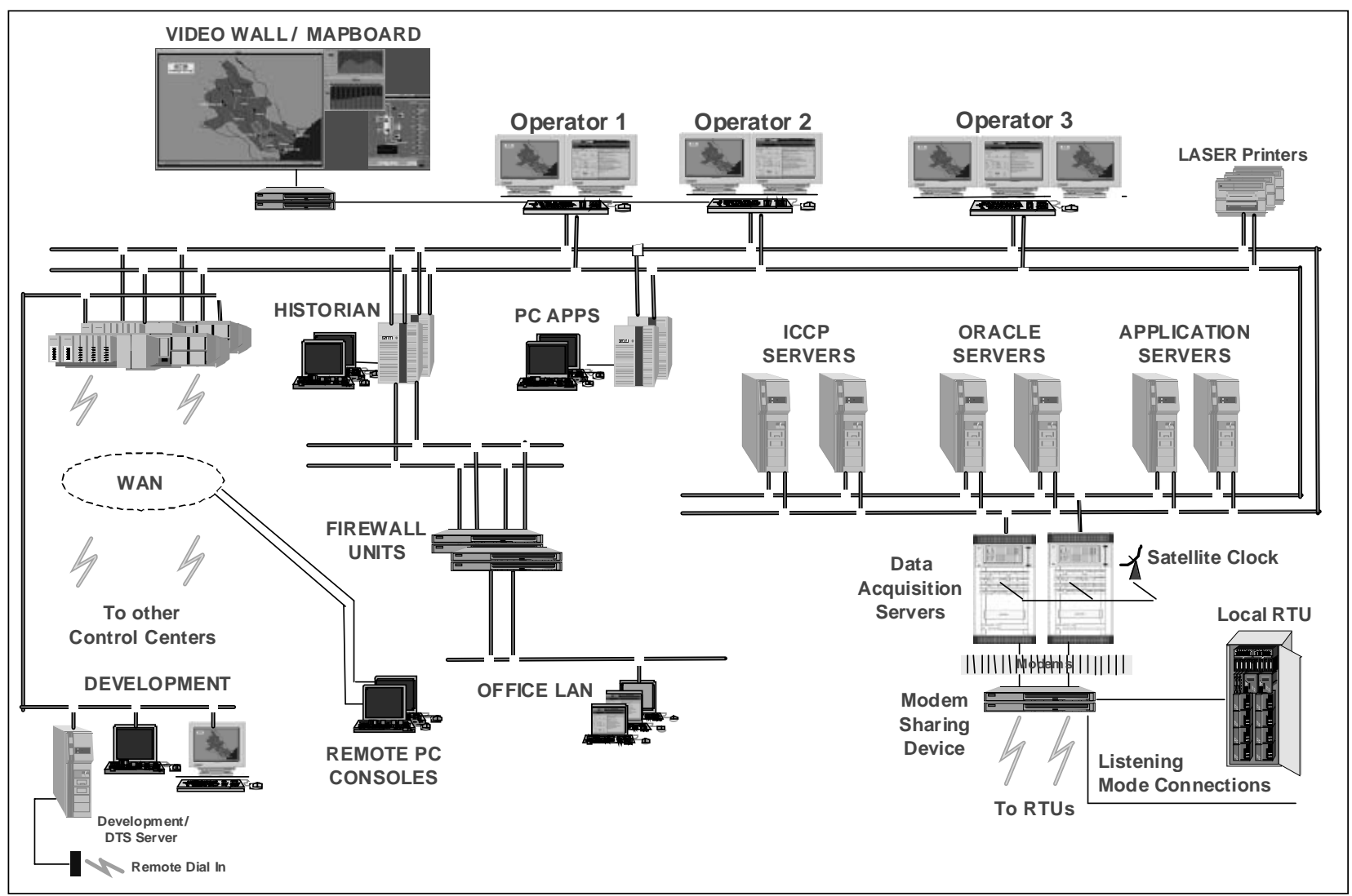

Fig. 3. Basic system Architecture 
software enable a host of application packages to be integrated and transforms the SCADA to be a very effective ERP tool for the electric utility.

A Distribution management system is required for the smooth operation of the network and to cater to the users demand for reliability and performance associated with high quality standardized proven designs. Nevertheless each utility operates its network differently and it requires functionalities, features, and control system capabilities often associated with tailor made designs. An integrated SCADA system for an urban city distribution system comprises of a host of distribution automation features.

\section{Focus of Distribution Management}

\section{First Priority - Control}

It is apparent from the majority of recent DMS implementation and surveys that been completed that implementation of remote control provides the fastest and greatest benefits to distribution operations.

Therefore, the first priority of any DMS is the real-time system control provided by the SCADA. The depth of this control is dependent on economics i.e. the largest payback is at the substation level (SA and traditional control through RTUs) down to the MV feeder circuit level. The next level is extended control or feeder automation (FA) outside the $\mathrm{HV} / \mathrm{MV}$ substations. The economic penetration of FA is determined by the Automation Intensity Level (AIL), which is defined as the percentage of all switches below the primary substation that will be automated and placed under SCADA control. The AIL produces a certain level of system performance in terms of reduction in average outage duration.

The AIL verses network performance level improvement comparison results in deciding the automation expenditure which is one of diminishing returns as shown in the figure 4 below for a typical feeder.

AIL is a key defining parameter of not only volume and performance improvement, but geographical location of the preferred location for automation. It must be set at the outset of any DMS project including FA. In summary the following levels of Depth of Control (DOC) can be defined.

DOC Level 1: Sub-transmission down to the grid (primary) substations including the medium voltage feeder circuit breakers within the substations

\section{Outage vs Number of Sections Automated}

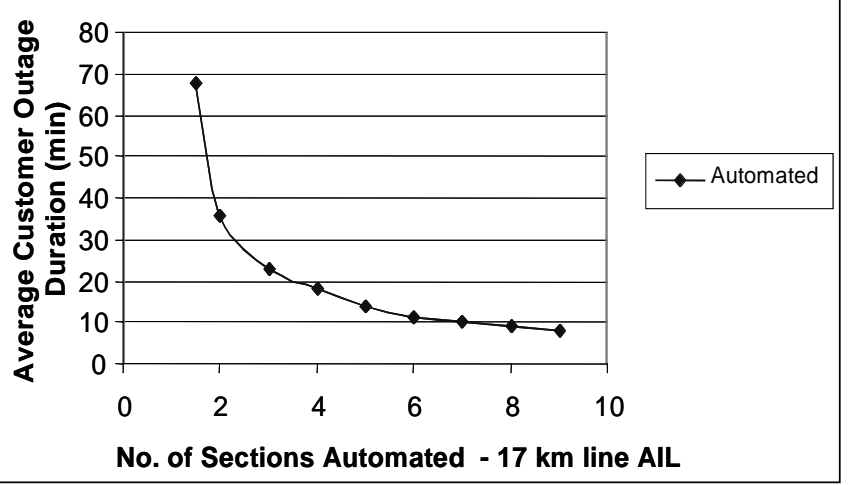

Fig. 4. Outage vs. Number of Sections Automated (AIL)

DOC Level 2: Level 1 extended to remotely operate (Feeder Automation) the switching and volt/var control devices along the distribution feeders outside the substation.

DOC Level 3: Including an element of control of low voltage networks. This level has not so far been justified by utilities however those with significant LV networks are viewing such extensions as the next areas for operational improvement.

\section{Second Priority - Control Room Management}

The second priority is to be able to manage the entire MV network of combined remotely controlled switches (10-15\% max) and the remaining manually operated devices. This is accomplished by the Control Room Management function, which employs a connectivity model of the entire electrical network, which can be viewed graphically. The diagram can be dressed manually to show changes in manually operated devices, as they are implemented by field crews, the application of tags to show areas of work or restrictions and also temporary network changes. SCADA derived information is displayed in real-time.

Unconstrained decision support decision support tools form the core of DMS advanced applications. They are less data dependent of the two categories (unconstrained and constrained) and rely purely on the analysis of network topology to provide operator support for all connectivity based analysis without considering network capacity line and load parameters and operating constraints. Such applications include

1) Dynamic Network Colouring

2) Switch Order Planning and Execution 
3) Fault Location, Isolation and System Restoration (FLISR)

They provide large incremental benefits without the need to assemble difficult to obtain data on network parameters and loads.

\section{Third Priority - Outage Management (Trouble Call Management)}

Different approaches to Outage Management have been taken in the industry and it is worth discussing these in order to recommend an appropriate solution for the Utility network.

Utilities with very limited penetration of real-time control but good customer and network records use a Trouble call approach, whereas those with good real-time systems and extended control were able to use direct measurements from automated devices. The former solution is prevalent in the USA for primary networks (MV- medium voltage) where distribution primary substations are smaller and except for large down town networks the low voltage (secondary) feeder system is limited with on the average between 6 and 10 customers being supplied from one distribution transformer. This system structure makes it easier to establish the customer-network link, a necessity for trouble call management systems if outage management is to yield any realistic results.

In contrast European systems with very extensive secondary systems (up to 400 consumers per distribution transformer) concentrated on implementing SCADA control thus any MV fault would be cleared and knowledge of the affected feeder known before any customer calls could be correlated. A trouble call approach to be truly affective would have to operate from the LV system where establishing the customer network link is more challenging. In these cases trouble call response was aimed at maintaining customer relation as a priority over fault location which is achieved through a combination of system monitoring applications (SCADA, FA) and advanced applications.

DMSs are now combining the best of these approaches to achieve real-time solutions on the network with customeroriented feedback. As a result of the two approaches two similar but different architectures have evolved.

\section{Geographical Information System (GIS) Centric.}

This is based on an extension of the GIS based geographical distribution network connectivity model and has been promoted by suppliers of GIS software as an additional GIS application. The geographical relation between the distribution network and the customer service entrance can be developed as part of the data of mains records. This with the addition of a link with the customer name and address from the Customer Information System (CIS) records provides the customer network link information and enables all geographically related tasks to be managed from essentially one application. The frequency of maintenance requirements of this link are relatively slow and can be satisfied by a batch transaction. The only missing data is the status of any circuit element which maybe operated under protection, SCADA or by the field crews directed from the control room. This information and thus the link to the GIS data model must operate at SCADA transaction speeds. In practice the GIS database were not designed for such transactional speeds and suffered from unacceptable performance when there was a significant penetration of real-time control. The performance was improved by moving the connectivity model onto a separate server mirroring any related changes to the GIS database; however the fast data link with SCADA still has to be maintained with the dedicated server. GIS Centric solutions have been implemented where very limited penetration of real-time control exists and the control room management function is in the majority GIS based. Thus all network data changes and extensions are within the frequency of most GIS database amendments.

\section{SCADA Centric:}

The second solution has been developed where the outage management process is dominated by real-time information. This information will precede any customer's knowledge of an outage on the MV network and hence his trouble call. Thus the trouble call process is more to maintain customer relations and association with the outage during the isolation and restoration process. However for any fault on the LV network undetectable by the SCADA the trouble call will provide invaluable information about an LV outage in a similar manner to the GIS centric solution. SCADA centric solutions also use a separate server (redundancy optional) that is synchronized with the SCADA runtime database in real-time. Any changes made to the SCADA database through switch status changes or by the operator as a manual change (temporary network change or as a data engineering amendment) will immediately update the trouble call server. The less frequent changes to the customer network link and customer information are implemented as regular batch transactions through the SCADA data engineering amendment Mechanism. In this type of solution the LV 
model is usually kept at a simple parent child relationship allowing customers to be associated with a supply point which in turn is associated with a fuse on the LV bus bar of the customer substation /distribution transformer.

In conclusion both solutions have moved to using dedicated servers for the trouble call application to ensure adequate performance. It is the penetration level of automation and the desired detail of LV representation (connectivity) that is realistic to maintain that are the main issues. Any DMS implementation that will be adding significant feeder automation should consider a SCADA centric solution not only from the real-time aspects but due to the regular amendments to the DMS data base as the operator brings on-line automated MV switches. Also many distribution control centres operate with orthogonal schematic diagrams thus the only advantage of geographical diagrams is for crew management. Geographic background maps can be either loaded as an additional display or accessed directly from the GIS through modern cross navigational features now readily available in most DMSs.

\section{Fourth Priority - Constrained Decision support Applications -}

Constrained decision support is possible when the network loading duty levels can be determined through load flow and fault analysis of the system. Such applications require in addition to topology, accurate information on the network element descriptive parameters such as impedance, capacity and most difficult to obtain, loads. This analysis can be integrated into the unconstrained decision tools to test the network loading at each step. Typical decision tools that are available include;

1) Operator Load Flow.

2) Fault Level calculation for circuit breaker duty analysis.

3) Constrained Fault location, Isolation and System Restoration (FLISR).

4) Loss Minimization, optimal switching and load balancing.

5) Voltage Automatic Regulator (VAR Control).

6) Voltage Optimization.

They also provide important data for Condition Based Maintenance of Network Assets.

\section{Data Dependency and Sustainability}

All DMS implementations are dependent on certain levels of network data for both the dynamic (real-time) and the static devices. This data is continually changing and thus an efficient data engineering system must deliver changes to the "as-built" system rapidly and the real-time system must be able to accommodate the operator implementing permanent changes in a timely manner on the run-time system. The more ambitious the decision support tools the greater the data dependency and resources to sustain the applications.

Incremental Economic Benefit and Data Complexity / Sustainability Index against data requirements for different decision tool applications.

The above discussion highlights some of the issues that must be resolved as part of the DMS implementation strategy and requirements to avoid creating a wish list that could generate a DMS outside both budgetary limits and operational practicalities.

\section{Basic SCADA Functionalities:}

This provides for real time monitoring and control of the power system network with the following functions.

1) Data acquisition - Furnishes Status information and measurands data to the operator

2) Control - Allows the operator to control the devices e.g., Circuit Breakers, Transformer Tap changers etc., from a remote centralized location.

3) Data processing - includes data quality and integrity check, Limit check, analog value processing etc.,

4) Tagging - Operator identifies any specific device and subjects to specific operating restrictions to prevent from unauthorized operations.

5) Alarms - Alerts the operator of unplanned events and undesirable operating conditions in the order of their severity and criticality.

6) Logging - Logs all operator entries, alarms and selected information.

7) Trending - Plots measurements on selected scale to give information on the trends eg., one minute, one hour etc.,

8) Historical Reporting - To save and analyze the historical data for reporting, typically for a period of 2 or more years and to archive. 


\section{Distribution Automation / Management functionalities}

The following functionalities form core of the Distribution Management system.

1) Integrated Volt Var Control

2) Network Geographic Information system

3) Load control and balancing / scheduling

4) Facilities Management

5) Trouble-call management systems

6) Operator Load Flow

7) Fault Localization, Isolation and System Restoration

\section{Integrated Volt Var control -}

One of the most important requirements of any distribution system is to provide a good voltage profile and the voltage within acceptable limits to the consumers to minimize distribution losses.

This is achieved by injecting the required reactive power at the appropriate locations in the network by installing shunt capacitors and voltage regulators on the distribution feeders and controlling them from the cen- tral location. The location and capacity of capacitor bank and voltage regulators can be decided using optimal minimal loss strategy while simultaneously satisfying the max/min voltage constraints on the feeder. The on-off switching commands for the capacitor banks and the raise-lower commands for voltage regulators will be issued from the central location. The analysis of the resulting situation is carried our by the system giving the revised voltage profile of the network.

\section{Network Geographic Information system-}

Network GIS is a core function for Distribution System Planning Studies and real time control / monitoring of Distribution Systems. The Distribution Systems are widely spread and have a large number of network elements. Further the System is dynamic and continuously changing in nature as new customers are added and new Facilities are installed every day. Each network element has a large attribute data. The Network GIS Package enables to capture all the details of the facilities and display them to provide a conceptual view of the location of the facility and also to monitor the maintenance, outages and the performance of the facility.

Figure5 shows the modular data base for real time application system.

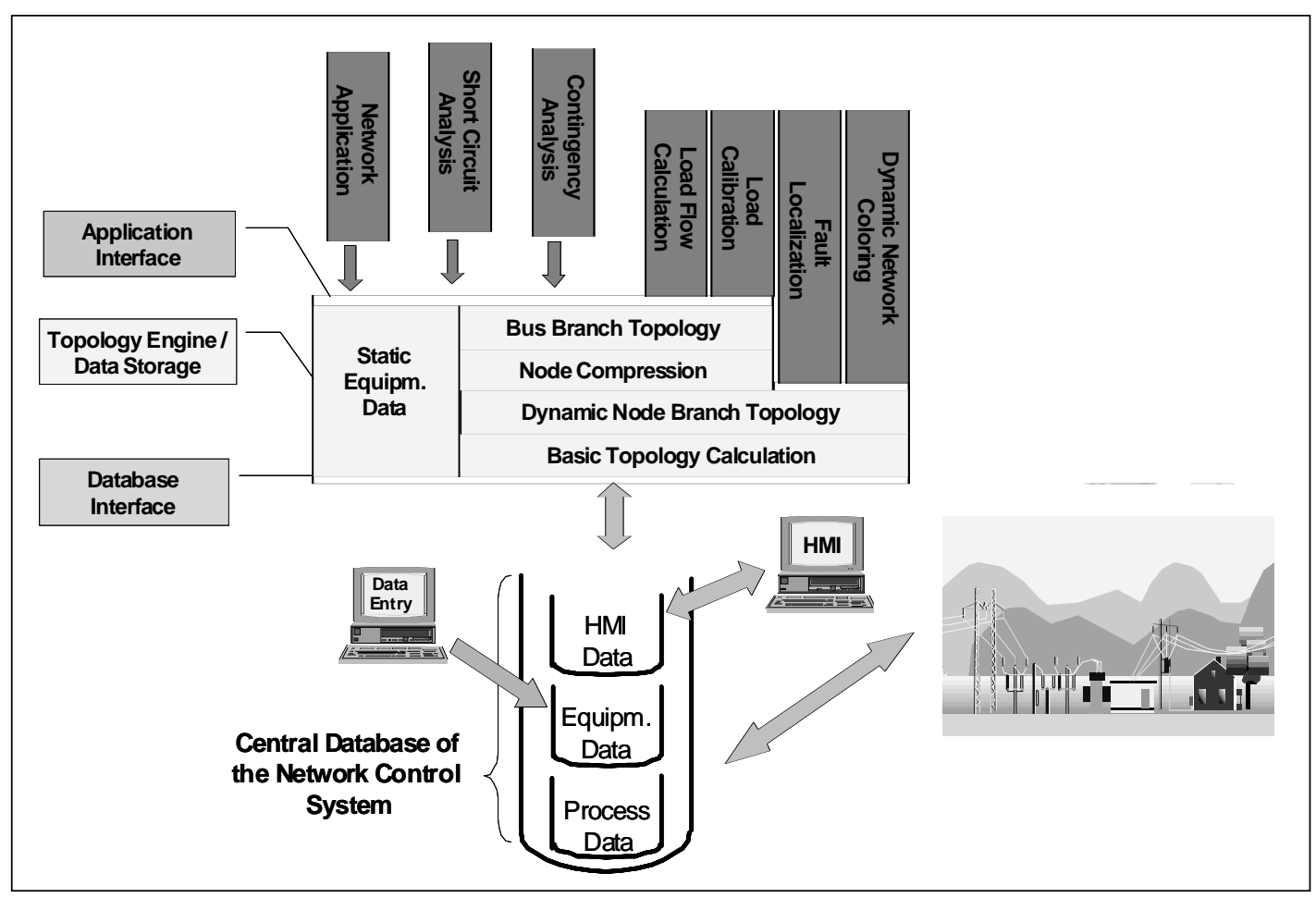

Fig. 5 Modular Database for Real time application systems 


\section{Load Control, Balancing \& Scheduling}

The aim of the function is to form minimum network loss configuration and also to balance the loads on the substations and feeders such that none of them are overloaded beyond their capacity. In respect of substation the continuous rating of power transformers and thermal loading limits of feeders is considered as the capacity of the substation. The network control function helps in better utilization of distribution facilities (transformer and feeder capacities) .This increases life expectancy of the equipment. The application determines switching status of the switches to ensure the best voltage profile and also minimal losses in the network while distributing the total load of the system among different feeders in the network such that none of the substations or feeders is overloaded. RTUs are provided at the substations for data acquisitions of the network, loading and status of the devices.

One of the requirements of the power system operation is to ensure that, generation and load demand are balanced under normal and emergency conditions. If at any time, the generation is less than the load demand, the frequency falls and may possibly lead to tripping of individual generator units due to loss of stability. This reduces the available power even more and may ultimately result in collapse of the electrical power system grid. This process takes place in a very short time and manual intervention by the load dispatcher is difficult. A total collapse of the network can be prevented by planned automatic load shedding program. The load shedding function not only prevents network collapses, but also increases the network stability and the power quality considerably. This application identifies the sheddable feeders in a systematic and scientific manner.

\section{Asset Management / Facilities Management}

The Asset Management / Facilities Management ( AM/FM) is generally an integral part of the Network GIS system. The AM/FM Package enables to capture all the details of the facilities and display them to provide a conceptual view of the location of the facility and also monitor the maintenance, outages and performance of the facility. The creation / population of AM/FM package requires a lot of effort on the part of utility, but it minimizes the cost of maintenance The AM/FM provides an exhaustive database of the equipment / devices / facilities owned or employed by the utility. The FM provides for recording the Technical Particulars of the facility, Periodical preventive maintenance carried out on the facility and outages occurred on the facility. The above data on the facilities enables the engineer to take effective steps to improve the availability of the facility and reduce the mean time between failures.

\section{Trouble call management system}

In a practical environment of power system operation, outages are inevitable. Consumers served from distribution system vary from homes with lighting load and small motors to complex loads, life saving hospitals, the chemical processing and hazardous industries. In the present day context, outages are not just an inconvenience but can also seriously affect production at all levels of commercial and industrial establishments. Hence when outages occur, the need for accurate and timely information is essential. Dispatch centers need to know precise outages locations and outages crew deployment in order to direct work crews to the location and restore supply.

The TCMS application analyses the calls entered and identifies the possible faulty device, keeps a track of crews available and directs the crews to the location for quick rectification of faults. The application provides timely information to the consumer on the status of rectification and also helps to reduce losses due to long interruptions and improve customer relations. Trouble calls are linked to the distribution transformer feeding the customer. The DTR in turn is linked with all upstream protective devices between the transformer and the station bus. Based on this information the TCM software identifies the problematic device and passes this information to the operators on duty for repair. Consumers are kept up to date on status of restoration of their individual outages. This outage and restoration information is retained for post-emergency analysis.

\section{Operator Load Flow - Balanced}

The purpose of the LF function is to provide the complete steady-state solution for a power system network for specified network conditions either in real-time or in study mode. In real time mode the LF function refines the output from the load calibration function by taking the voltage dependency of the load into account. In study mode the LF function can be used to study possible operating situations in order to facilitate the selection of control actions and network operation. In particular it enables the operator to examine the voltage distribution (magnitudes and angles) and the resulting quantities such as branch flows, injections, currents and loss- 
es corresponding to a set of operating conditions. All calculated values are limit checked and limit violations are flagged.

\section{Fault Localization, Isolation and System Restoration}

The FLISR-function gives the operator a hand during system disturbance by filtering the incoming system messages and supplying with diagnosis information and recommendations for remedial actions. The short circuit localization part of the FLISR-function helps the operator to deal with a short circuit swiftly and safely. The faulty region is narrowed down as much as possible so that the operator can take action to eliminate the failure.

The isolation and restoration part of the FLISR-function gives the operator a set of recommendations for remedial actions to help the operator to re-establish the power supply for as many customers as possible without wasting time.

The FLISR-function is designed as a background function doing its work with a minimum of operator interventions.

\section{Evaluation of Distribution Management System imple- mentation}

The audits of a typical Indian utility distribution network, its operating procedures and organization structure indicated that improvements can be made in the following areas by the implementation of a DMS facility. These improvements are mapped against DMS functions that produce the benefits. These operating business objectives are consolidated into eight categories

1. Improve reliability and quality at optimum cost

2. Improve Customer Relationship Management (CRM)

3. Better knowledge of network available transfer capacity.

4. Intelligent Load Shedding

5. Timely, up-to-date data on actual system state/performance available to all decision makers (management, planners and operators).

6. Coordinated documentation and information on network assets.

7. Streamline business and operating processes.

8. Reduction of all losses - technical and commercial.
Successful integration and automation projects require a strategic plan, or a blue print how and when various components of automation and integration architectures will be installed. Matching the needs of utilities with available technology is critical. Sometimes automation technology is not yet available to accomplish what utility needs, since it should have capability to integrate with older primary equipment and their proprietary protocols.

The next step is to develop a phased approach for implementation of integration and automation. This means that existing communication technology must be compared with what will be needed for the planned project and for future automation. The best measurement to use in assessing the success of any size automation project is the industry standard indices of reliability i.e.SAIDI, SAIFI and CAIDI. The evaluation of the application of Distribution Automation to the utility distribution system, must concentrate on economic solutions to the problems. Electric distribution utility frequently asks the question; how we can best move toward automation. Given the nature of Investment and expectation it is imperative to plan the automation in a phased manner so that best result is achieved.

The two major aspects are economical and technical which need to be evaluated so as to provide quality power to customers and the best utilization of investment. An approach to the phased automation provides the utility a framework for building new substations or modernizing existing substation e.g. in the successful integration substation there will be smaller control panels, reduced wiring and elimination of conventional displays as computers replace mechanical device. Every enterprise wide integration and automation projects pay off in one to three years, but utilities have to access the quantitative result immediately.

\section{Description of developed Prototype model}

\section{Hardware model.}

The application of the embedded system prototype model is to monitor the conditions of the devices/equipments such as transformer in the sub-station. The circuit comprises of 89C51 microcontroller, ADC 0808, ULN 2003A, LM 7805, LM35, MAX232, RS232, step down transformer, bridge rectifier, cooling fan, electromagnetic relay, LCD, DC motor, 555 timer, crystal oscillator and variable POT as shown in figure 6 and the functional diagram in figure7. 


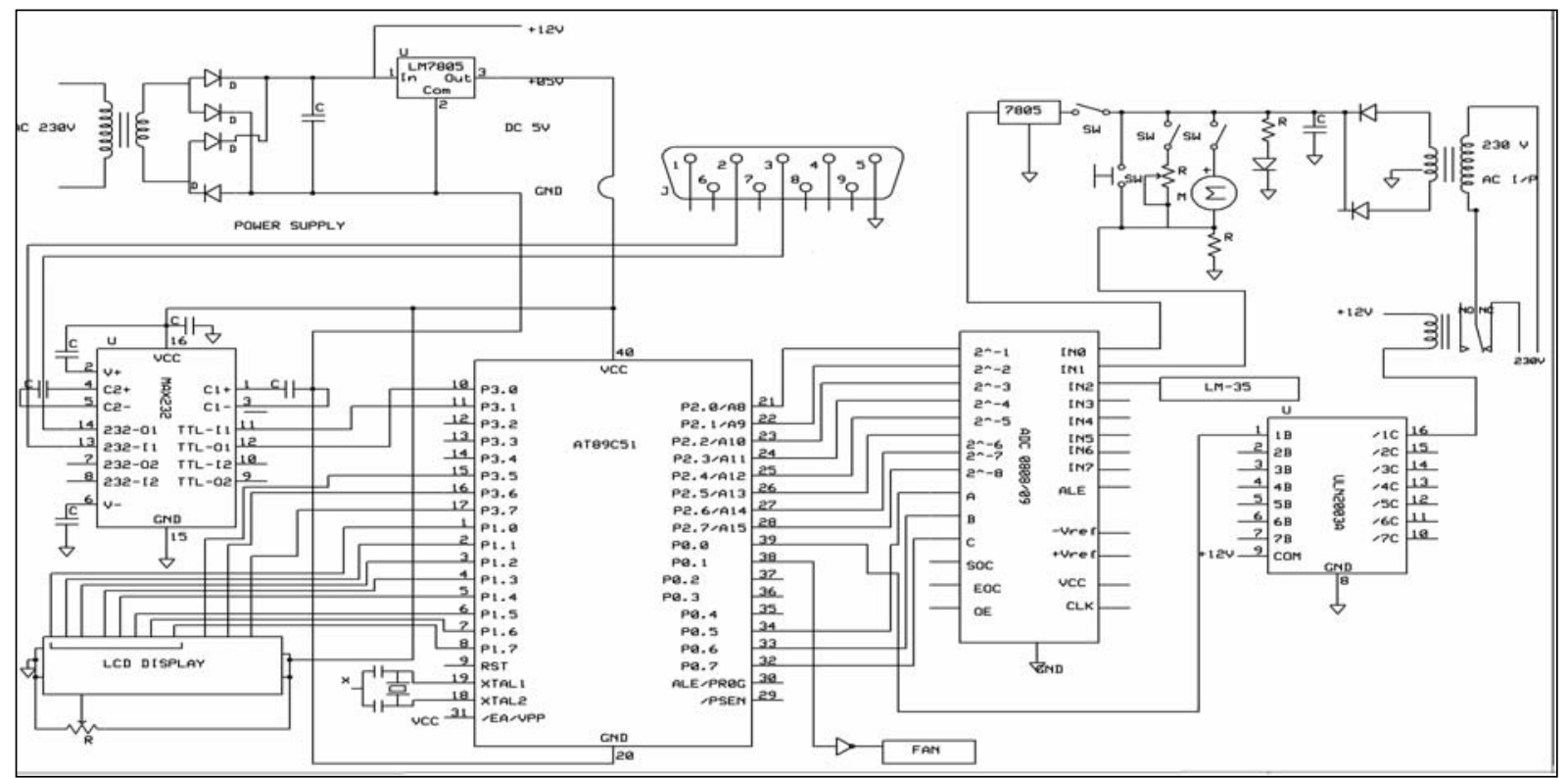

Fig.6. Hardware Diagram of the Model

The Hardware model has two sections the substation part and the controller part. When the substation is in ON condition the ADC continuously receives the analogue input value and delivers digital output to the controller by successive approximation. The microcontroller is also connected to the
LCD to view the status conditions of the power system and to the relay driver circuit to open its contacts whenever a fault such as open circuit or short circuit occurs.

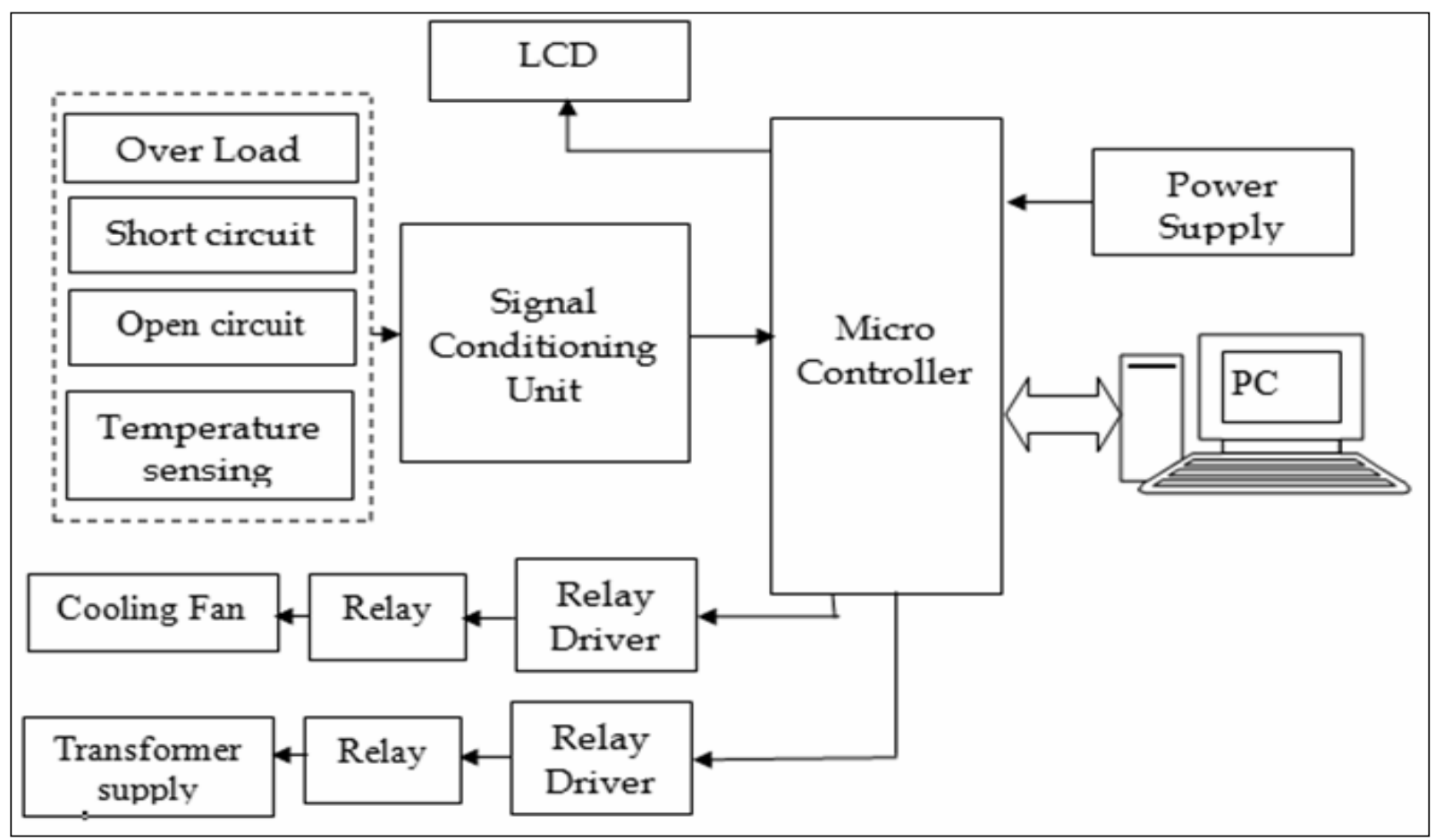

Figu. 7. The functional Block Diagram 
Similarly the controllers do have a connection to the cooling fan in the sub-station which starts operating when the temperature of the transformer exceeds a preset value. This operation is accomplished by using a LM35 sensor. In addition to the LCD display the status condition of the system can also be visualized by interfacing it with a Personal computer using RS232 serial communication port. The voltage regulator LM7805 is employed to supply a fixed output voltage of $5 \mathrm{~V}$ to controller, ADC, LCD and 12V to ULN2003A, MAX 232 and cooling fan The circuit diagram of figure 6 comprises of 89C51 microcontroller, analog to digital converter (0808), MAX232, voltage regulator (LM7805), temperature sensor (LM35) and liquid crystal display (LCD).The Vcc pin of microcontroller is connected to the $+5 \mathrm{~V}$ output of voltage regulator (LM7805). The digital output of the ADC is given to the port 2 of the microcontroller. Channel select pins A, B, $\mathrm{C}$ are connected to the port $\mathrm{p} 0.5, \mathrm{p} 0.6, \mathrm{p} 0.7$ respectively. The port p0.0 has a connection to the pin number 1 of relay driver circuit (ULN 2003A). The eight pins of port 1 is connected to the LCD display. Similarly the pin number 18 and 19 of the controller has a connection to crystal oscillator. The port p0.1 is connected to the cooling fan and port p3.0 and p3.1 to the IC MAX 232.The MAX 232 consists of 4 capacitors connected in parallel. MAX 232 is provided for serial data transmission with the port provided. A $+12 \mathrm{~V}$ supply is given to the pin number 9 of ULN 2003A.The pin IN2 of the ADC 0808 is connected to the temperature sensor LM35.The analog input is given to the pin IN0, IN1 of the ADC 0808. Bridge rectifier is connected across the secondary of the step down transformer (i.e., four diodes) to convert the step down AC into a pulsating DC of $12 \mathrm{~V}$. A capacitor filter is connected across it to make into a pure DC.

\section{Software Description}

\section{Keil C programming}

The use of Keil uVision2 Integrated development environment of a 'C' based, Micro converter application soft ware, the Keil 8052 compiler package which includes uVision2 an Integrated Development Environment (IDE) along with all the utilities which may need to create embedded application programs for the micro converter family. An evaluation copy of the Keil C51 developers kit (PK51) is available as part of micro converter tools chain or directly from the Keil software. This evaluation copy will create applications that are 2Kbytes and smaller. It is used to create and test the target programs and also to evaluate the Keil environment. Another powerful feature of the uVision2 IDE is that it allows you to run your code in a micro converter specific simulation environment. To start a simulation session you simply click on the 'Start/Stop Debug Session' option available from the 'Debug' pull-down menu. Alternatively you can press $<$ ctrl+F7> or the 'Debug' icon available in the 'File' toolbar (Kenneth 1996 and Muhammad 1999).

\section{Configuring the Performance Analyzer}

First configure the performance analyzer (PA). This is done simply by right clicking on the PA and selecting the 'Setup PA' option. In the resultant dialog box, double click on each function symbol and press 'Define' in turn. Once the operation is completed for each function symbol press 'close' and the PA window should be configured. To start the simulation, click on the GO icon in the DEBUG toolbar. Immediately it will see the serial $\mathrm{I} / \mathrm{O}$ window being updated with the data being transmitted by the micro converter UART in this simulation session and it should also note that the performance analyzer is being updated to reflect where code execution is spending most of its time.

\section{Simulating an External Interrupt}

An external interrupt 0 can be simulated by clearing the $\mathrm{p} 3.2$ bit in the port window. In response to the simulated interrupt, the 16 values in the RAM are transmitted via the UART and code execution will not return from the Interrupt Service Routine (ISR) until it reset the p3.2. Again before it clear the p3.2 bit it should note that the performance analyzer changes to reflect how the code is now waiting in the INTO ISR.

\section{Visual Basic for Application}

Programming can be an enormously complex and difficult activity or it can be quite straight forward. In either case, the basic programming concepts remain the same. This is an introduction to a handful of programming constructs that applies to any "third generation" language not only visual basic for applications.

\section{Interacting with the Interpreter}

Access provides two ways of interacting with the VBA language. The most useful of these is through saved modules that Contain VBA procedures. These procedures can be run to do interesting things like process transactions against master tables, Provide sophisticated error checking and so on. The second way to interact with VBA is directly through the interpreter. Interpreted languages are 
to experiment with since you can invoke the interpreter at any time, type in a command, and watch it execute.

\section{Interpreted and Compiled Languages}

VBA is an interpreted language. In interpreted languages, each line of the program is interpreted and executed when the program is run. Other languages are compiled, meaning that the original program is translated and saved into a file of machine languages commands. This executable file is run instead of the source code. Predictably, compiled languages run much faster than languages. However, interpreted languages are typically easier.

\section{Developed Program}

\#include <reg51.h>

\#include"lcd_blue.h"

Void write (unsigned char *);

Void adcconv (unsigned char );

Void txs (unsigned char);

Void msdelay ();

unsigned char $\mathrm{i}$;

sfr adc $=0 \mathrm{xa} 0$;

sfr $r f t x=0 x 80$;

sbit fan $=\mathrm{P} 0 \wedge 0$;

sbit pin0 $=\mathrm{P} 3 \wedge 4$;

sbit $\mathrm{a}=\mathrm{P} 3 \wedge 5$;

sbit $b=P 3 \wedge 6$;

sbit $\mathrm{c}=\mathrm{P} 3 \wedge 7$;

unsigned char pin;

void main()

\{

// $\quad \mathrm{rftx}=0 \mathrm{x} 00$;

fan $=0$;

lcdinit();

lcdcmd(0x80);

write("FAULT INDICATION");

pin $0=0$;

lcdcmd(0xc0);

write("STATUS:");

adc $=0 \mathrm{xff}$;

while (1)

\{

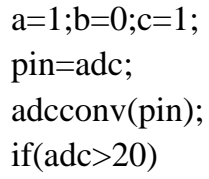

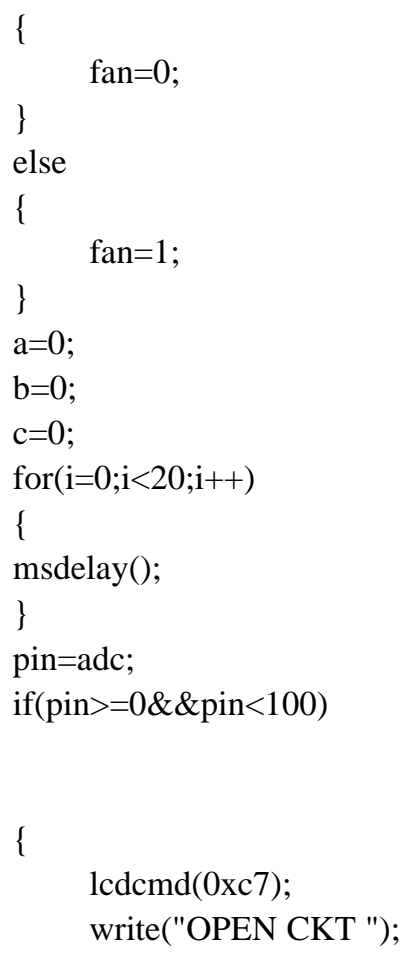




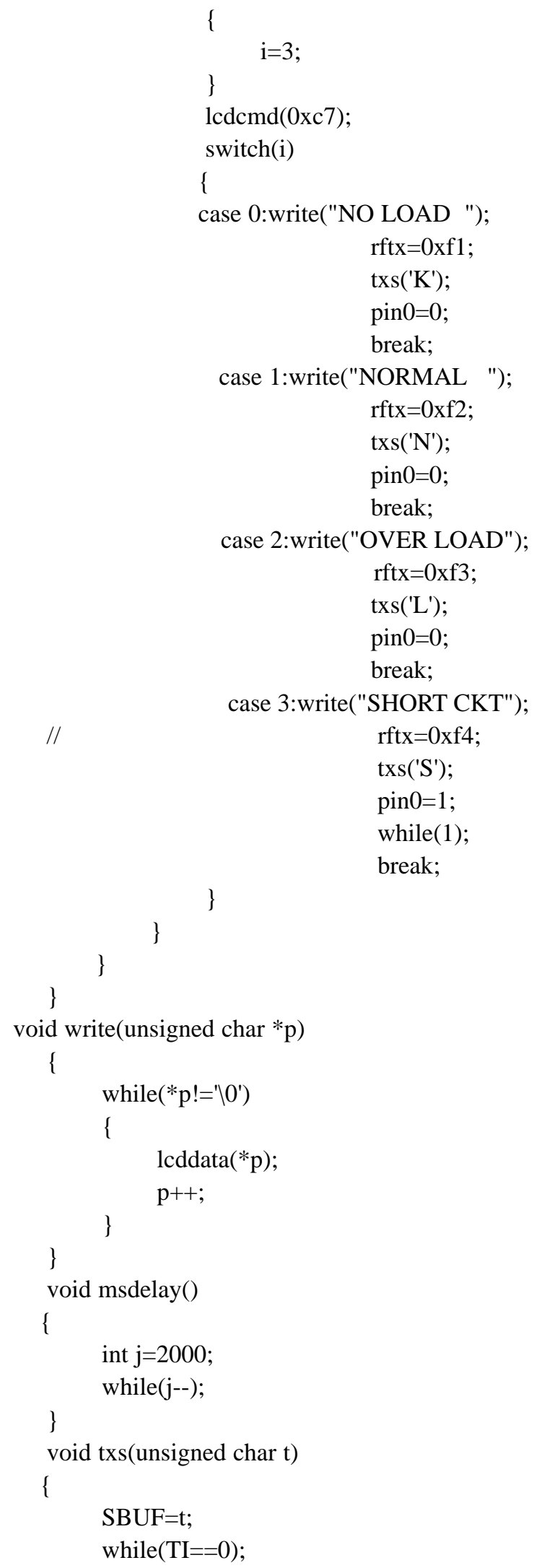

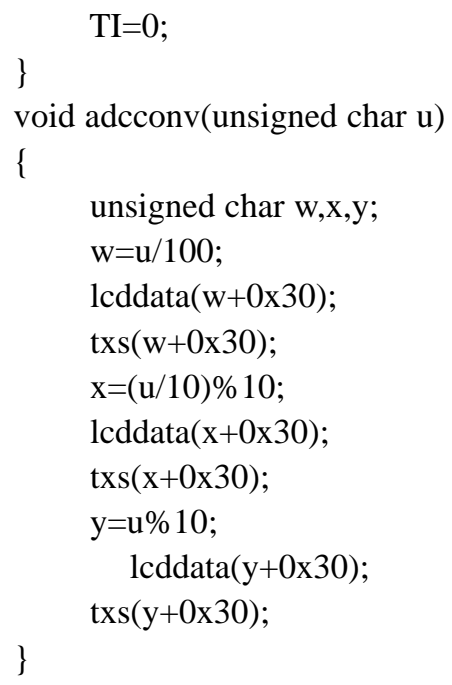

\section{Result and Discussion}

SCADA refers to a system that collects data from various sensors in the substation or in other remote locations and then sends this data to a central computer which manages and controls the system.

This novel hardware prototype model proved that the temperature sensor will continuously monitor the safe set value of the design temperature limits of the transformer and gives this input to the microcontroller through the interface circuit. Transformer is being monitored continuously for the overload for either exceeding the design values or in case of fault in the power system by the opening of the circuit. Microcontroller accepts the data in digital form to compare with set value for the satisfactory operation of the system and in case of abnormality to trip the whole unit or the corresponding fault unit.

In this model the successful monitoring of the conditions of the miniature substation represented in our prototype model as the normal load, over load, open circuit, short circuit and temperature are observed. When the above said conditions are altered in the power system the controller will break the corresponding device from the fault and thus ensures the safety of the components of the power system.

\section{Conclusion}

This is vividly evident that SCADA Power Distribution Automation Systems offer as a solution to improve power distribution efficiencies.

Also the indigenously developed sample prototype hardware and software model utilising the latest embedded technology 
innovation for SCADA Power Distribution Automation Systems will reliably perform the power system.

\section{References}

Ahmed M. M. and Soo W. L. (2008) (Malaysia), Development of Customized Low Voltage (LV) Distribution Automation System (DAS) for Effective Fault Operation and Fault Isolation, Proceeding (617) Power and Energy Systems.

Antila E., Heine P. and Lehtonen M. (2003) Economic Analysis of Implementing Novel Power Distribution Automation, CIGRE/IEEE PES International Symposium on Quality and Security of Electric Power Delivery Systems, Montreal, Canada, October 8-10.

Dragan P., Vladimir S. and Nenad K. (2001) Power Applications-A Powerful Tool for Distributed Network Management, CIRED Conference,Session Operation control and protection of supply systems, Amsterdam, pp 1-5.

Katic N., Strezoski V. and Popovic D. (2002) DMS Software Applications - a Powerful Tool for the New Challenges in Deregulated Power Distribution, Balkan Power Conference Proceedings Vol.2, pp.125-130, Belgrade, pp 125-130.

Kenneth J. Alya (1996) The 8051 microcontroller architecture, programming and application. 2nd edition. International Thompson publishers.
Muhammad A. M. (1999) The 8051 microcontroller and embedded systems prentice hall.

Nenad Katic (1995) The Application of the New Technologies as a Solution for Present Power Distribution Problems, Thessaloniki, Greece, pp 1-7.

Nenad K. and Vladimir S. (1998) M V Network Power Analysis Based on State Estimation, DA/DSM Europe Distributech, London, England, pp 1-8.

Roytelman I. and Shahidehpour S. M. (1993) Department of Electrical and Computer Engineering Illinois Institute of Technology Chicago, Illinois 60616, state estimation for electric power distribution systems in quasi real-time conditions, IEEE Transactions on Power Delivery, Vol. 8, No. 4, October.

Successful Integration and Automation realize on Strategic Plans: John McDonald KEMA

Vladimir Katie (1995) Nenad Katie: Investigation of Harmonic Pollution in Low-Voltage Distribution Network, Distributed Automation and Demand Side Management, Europe Conference, Rome, Italy, pp 113

Received : April 15, 2009;

Accepted : September 09, 2009 